\title{
Pointwise approximation for a type of Bernstein-Durrmeyer operators
}

\section{Guofen Liu}

\section{"Correspondence:} liugf2003@163.com College of Mathematics and Information Science, Hebei Normal University, Shijiazhuang, 050024, People's Republic of China Hebei Key Laboratory of Computational Mathematics and Applications, Shijiazhuang, 050024, People's Republic of China

\begin{abstract}
We give the direct and inverse approximation theorems for a new type of Bernstein-Durrmeyer operators with the modulus of smoothness.

MSC: $41 \mathrm{~A} 25 ; 41 \mathrm{~A} 27 ; 41 \mathrm{~A} 36$

Keywords: Bernstein-Durrmeyer type operator; modulus of smoothness; $K$-functional; direct and inverse approximation theorems
\end{abstract}

\section{Introduction}

Durrmeyer [1] introduced the integral modification of the well-known Bernstein polynomials given by

$$
D_{n}(f, x)=(n+1) \sum_{k=0}^{n} p_{n, k}(x) \int_{0}^{1} p_{n, k}(t) f(t) d t
$$

where $p_{n, k}(x)=\left(\begin{array}{l}n \\ k\end{array}\right) x^{k}(1-x)^{n-k}$. Derriennic [2] established some direct results in ordinary and simultaneous approximation for Durrmeyer operators. Then Durrmeyer type operators were studied widely [3-5]. Recently Gupta et al. [6] considered a family of Durrmeyer type operators:

$$
P_{n, m}(f, x)= \begin{cases}n \sum_{k=1}^{n} p_{n, k}(x) \int_{0}^{1} p_{n-1, k-1}(t) f(t) d t+p_{n, 0}(x) f(0), & m=0 \\ \frac{n^{m}}{(n+m-1) \underline{\underline{m-1}}} \sum_{k=0}^{n-m} p_{n-m, k}(x) \int_{0}^{1} p_{n+m-1, k+m-1}(t) f(t) d t, & m>0\end{cases}
$$

where $m, n \in N_{0}$ with $m \leq n$ and for any $a, b \in N_{0}, a^{\underline{b}}=a(a-1) \cdots(a-b+1), a^{\underline{0}}=1$ is the falling factorial; and we get the rate of convergence for these operators for a function having derivatives of bounded variation and the result in the simultaneous approximation. In the present note our main aim is to get the direct and inverse approximation theorem for this type of operators. Here we shall utilize modulus of smoothness and $K$-functional as the tools, which are defined by [7]

$$
\begin{aligned}
& \omega_{\varphi^{\lambda}}^{2 r}(f, t)=\sup _{0<h \leq t} \sup _{x \pm h r \varphi^{\lambda}(x) \in[0,1]}\left|\triangle_{h \varphi^{\lambda}(x)}^{2 r} f(x)\right|, \\
& K_{\varphi^{\lambda}}\left(f, t^{2 r}\right)=\inf _{g^{(2 r-1)} \in A \cdot C \cdot C_{\text {loc }}}\left\{\|f-g\|+t^{2 r}\left\|\varphi^{2 r \lambda} g^{(2 r)}\right\|\right\},
\end{aligned}
$$

O2014 Liu; licensee Springer. This is an Open Access article distributed under the terms of the Creative Commons Attribution License (http://creativecommons.org/licenses/by/2.0), which permits unrestricted use, distribution, and reproduction in any medium, provided the original work is properly cited. 
where $\varphi(x)=\sqrt{x(1-x)}, 0 \leq \lambda \leq 1, r \in N$. It is well known that $\omega_{\varphi^{\lambda}}^{2 r}(f, t) \sim K_{\varphi^{\lambda}}\left(f, t^{2 r}\right)$, where $a \sim b$ means that there exists some constant $C>0$ such that $C^{-1} b \leq a \leq C b$. We denote $M_{n, m}(f, x)=\frac{(m+n) \underline{m}}{n^{\underline{m}}} P_{n, m}(f, x)$ and state our main results as follows.

Theorem 1 For $f \in C[0,1], 0<\lambda<1, \varphi(x)=\sqrt{x(1-x)}$, one has

$$
\left|M_{n, m}(f, x)-f(x)\right| \leq C\left(\omega_{\varphi^{\lambda}}^{2}\left(f, \frac{1}{\sqrt{n}}\right)+\frac{1}{n}\|f\|\right) .
$$

Theorem 2 Let $f \in C[0,1], 0 \leq \lambda \leq 1, \varphi(x)=\sqrt{x(1-x)}, \delta_{n}(x)=\max \left\{\varphi(x), \frac{1}{\sqrt{n}}\right\}, r \in N, 0<$ $\alpha<2 r$, for $m>0$, and from

$$
\left|M_{n, m}(f, x)-f(x)\right|=O\left(\left(n^{\frac{1}{2}} \delta_{n}^{1-\lambda}(x)\right)^{\alpha}\right),
$$

we get $\omega_{\varphi^{\lambda}}^{2 r}(f, t)=O\left(t^{\alpha}\right)$.

Throughout this paper $\|\cdot\|=\|\cdot\|_{\infty}$ and $C$ denotes a positive constant independent of $n$ and $x$ not necessarily the same at each occurrence.

\section{Lemmas}

To prove the above theorems we need the following lemmas. First we define the moments, for any $s \in N_{0}, T_{n, m, s}(x)=M_{n, m}\left((t-x)^{s}, x\right)$.

Lemma 3 ([6]) The following claims hold.

(1) For any $s, m \in N_{0}, x \in[0,1]$, the following recurrence relation is satisfied:

$$
\begin{aligned}
(n+m+s+1) T_{n, m, s+1}(x)= & x(1-x)\left[T_{n, m, s}^{\prime}(x)+2 s T_{n, m, s-1}(x)\right] \\
& +[(s+m)-x(1+2 s+2 m)] T_{n, m, s}(x),
\end{aligned}
$$

where for $s=0$, we denote $T_{n, m,-1}(x)=0$.

(2) For any $m \in N_{0}$ and $x \in[0,1]$,

$$
\begin{aligned}
& T_{n, m, 0}(x)=1, \quad T_{n, m, 1}(x)=\frac{m-x(1+2 m)}{n+m+1}, \\
& T_{n, m, 2}(x)=\frac{2 n x(1-x)+m(1+m)-2 m x(2 m+3)+2 x^{2}\left(2 m^{2}+4 m+1\right)}{(n+m+1)(n+m+2)} .
\end{aligned}
$$

(3) For any $s, m \in N_{0}, x \in[0,1], T_{n, m, s}(x)=O\left(n^{-[(s+1) / 2]}\right)$.

Remark For $n$ sufficiently large and $x \in(0,1)$, it can be seen from Lemma 3 that

$$
\frac{x(1-x)}{n} \leq T_{n, m, 2}(x) \leq \frac{C x(1-x)}{n},
$$

for any $C>2$.

Lemma 4 For $f(x) \in C[0,1], \varphi(x)=\sqrt{x(1-x)}, \delta_{n}(x)=\max \left\{\varphi(x), \frac{1}{\sqrt{n}}\right\}, 0 \leq \lambda \leq 1, r \in N$, $m>0$, we have

$$
\left|\varphi^{2 r \lambda}(x) M_{n, m}^{(2 r)}(f, x)\right| \leq C n^{r} \delta_{n}^{2 r(\lambda-1)}\|f\| .
$$


Proof To complete the proof we consider two cases of $x \in E_{n}=\left[\frac{1}{n}, 1-\frac{1}{n}\right]$ and $x \in E_{n}^{c}=$ $\left[0, \frac{1}{n}\right] \cup\left[1-\frac{1}{n}, 1\right]$.

For $x \in E_{n}^{c}, \varphi^{2}(x) \leq \frac{C}{n}, \delta_{n}^{2}(x) \sim \frac{1}{n}$. Using

$$
M_{n, m}^{(2 r)}(f, x)=\frac{(n-m) !}{(n-m-2 r) !} \sum_{k=0}^{n-m-2 r} p_{n-m-2 r, k}(x) \triangle^{2 r} a_{k}(n)
$$

where $a_{k}(n)=(n+m) \int_{0}^{1} p_{n+m-1, k+m-1}(t) f(t) d t, \Delta a_{k}(n)=a_{k+1}(n)-a_{k}(n), \Delta^{r} a_{k}(n)=$ $\triangle\left(\triangle^{r-1} a_{k}(n)\right)$; and $\left|\triangle^{2 r} a_{k}(n)\right| \leq C\|f\|, \frac{(n-m) !}{(n-m-2 r) !} \leq(n-m)^{2 r}<n^{2 r}$, one has

$$
\left|\varphi^{2 r \lambda}(x) M_{n, m}^{(2 r)}(f, x)\right| \leq C n^{-r \lambda} n^{2 r}\|f\| \leq C n^{r} \delta_{n}^{2 r(\lambda-1)}\|f\| .
$$

For $x \in E_{n}, \delta_{n}(x) \sim \varphi(x)$. From [7] we have

$$
M_{n, m}^{(2 r)}(f, x)=\varphi^{-4 r}(x) \sum_{i=0}^{2 r} Q_{i}^{B}(x, n) n^{i} \sum_{k=0}^{n-m} p_{n-m, k}(x)\left(\frac{k}{n-m}-x\right)^{i} a_{k}(n),
$$

where $Q_{i}^{B}(x, n)$ a polynomial in $n \varphi^{2}(x)$ of degree [(2r-i)/2] with non-constant bounded coefficients. Therefore,

$$
\left|\varphi^{-4 r}(x) Q_{i}^{B}(x, n) n^{i}\right| \leq C\left(\frac{n}{\varphi^{2}(x)}\right)^{r+\frac{i}{2}}
$$

By Holder's inequality we get

$$
\sum_{k=0}^{n-m} p_{n-m, k}(x)\left(\frac{k}{n-m}-x\right)^{i} \leq\left(\sum_{k=0}^{n-m} p_{n-m, k}(x)\left(\frac{k}{n-m}-x\right)^{2 i}\right)^{\frac{1}{2}} \leq C n^{-\frac{i}{2}} \varphi^{i}(x) .
$$

Consequently $\left|\varphi^{2 r}(x) M_{n, m}^{(2 r)}(f, x)\right| \leq C n^{r}\|f\|$, hence

$$
\left|\varphi^{2 r \lambda}(x) M_{n, m}^{(2 r)}(f, x)\right|=\varphi^{2 r(\lambda-1)}(x)\left|\varphi^{2 r}(x) M_{n, m}^{(2 r)}(f, x)\right| \leq C n^{r} \delta_{n}^{2 r(\lambda-1)}\|f\| .
$$

From (2.3) and (2.4), (2.2) holds.

Lemma $5 \operatorname{For~}^{(2 r-1)}(x) \in A$.C. ${ }_{\text {loc }},\left\|\varphi^{2 r \lambda} f^{(2 r)}\right\|<\infty, m>0$, we have

$$
\left|\varphi^{2 r \lambda}(x) M_{n, m}^{(2 r)}(f, x)\right| \leq C\left\|\varphi^{2 r \lambda} f^{(2 r)}\right\| .
$$

Proof From $p_{n-m, k}^{(2 r)}(x)=\frac{(n-m) !}{(n-m-2 r) !} \sum_{i=0}^{2 r}(-1)^{i}\left(\begin{array}{c}2 r \\ i\end{array}\right) p_{n-m-2 r, k-(2 r-i)}(x)$, we have

$$
\begin{aligned}
& M_{n, m}^{(2 r)}(f, x) \\
& \quad=(n-m) \sum_{k=0}^{n-m} p_{n-m, k}^{(2 r)}(x) \int_{0}^{1} p_{n+m-1, k+m-1}(t) f(t) d t \\
& \quad=\frac{(n-m)(n-m) !}{(n-m-2 r) !} \sum_{k=0}^{n-m-2 r} \sum_{i=0}^{2 r}(-1)^{i}\left(\begin{array}{c}
2 r \\
i
\end{array}\right) p_{n-m-2 r, k-(2 r-i)}(x) \int_{0}^{1} p_{n+m-1, k+m-1}(t) f(t) d t
\end{aligned}
$$




$$
\begin{aligned}
= & \frac{(n-m)(n-m) !}{(n-m-2 r) !} \sum_{k=0}^{n-m-2 r} p_{n-m-2 r, k}(x) \int_{0}^{1} \sum_{i=0}^{2 r}(-1)^{i}\left(\begin{array}{c}
2 r \\
i
\end{array}\right) p_{n+m-1, k+m+2 r-i-1}(t) f(t) d t \\
= & \frac{(n-m)(n-m) !}{(n-m-2 r) !} \frac{(n+m-1) !}{(n+m-1+2 r) !} \\
& \times \sum_{k=0}^{n-m-2 r} p_{n-m-2 r, k}(x) \int_{0}^{1} p_{n+m+2 r-1, k+m+2 r-1}(t) f^{(2 r)}(t) d t .
\end{aligned}
$$

Let $I=(n-m) \sum_{k=0}^{n-m-2 r} p_{n-m-2 r, k}(x)\left|\int_{0}^{1} p_{n+m+2 r-1, k+m+2 r-1}(t) f^{(2 r)}(t) d t\right|$. For $0 \leq \lambda \leq 1$ one has

$$
\begin{aligned}
& \varphi^{2 r \lambda}(x) I \\
& \quad \leq\left\|\varphi^{2 r \lambda} f^{(2 r)}\right\| \varphi^{2 r \lambda}(x) \sum_{k=0}^{n-m-2 r} p_{n-m-2 r, k}(x)(n-m) \int_{0}^{1} p_{n+m+2 r-1, k+m+2 r-1}(t) \varphi^{-2 r \lambda}(t) d t \\
& \quad \leq\left\|\varphi^{2 r \lambda} f^{(2 r)}\right\|\left(\sum_{k=0}^{n-m-2 r} p_{n-m-2 r, k}(x) \varphi^{2 r}(x)(n-m) \int_{0}^{1} p_{n+m+2 r-1, k+m+2 r-1}(t) \varphi^{-2 r}(t) d t\right)^{\lambda} .
\end{aligned}
$$

Noting that

$$
\begin{aligned}
& p_{n-m-2 r, k}(x) \varphi^{2 r}(x)=\frac{(n-m-2 r) !(k+r) !(n-m-k-r) !}{k !(n-m-2 r-k) !(n-m) !} p_{n-m, k+r}(x)=: \alpha_{n, k} p_{n-m, k+r}(x) \\
& p_{n+m+2 r-1, k+m+2 r-1}(t) \varphi^{-2 r}(t) \\
& \quad=\frac{(n+m+2 r-1) !(k+m+r-1) !(n-k-r) !}{(k+m+2 r-1) !(n-k) !(n+m-1) !} p_{n+m-1, k+m+r-1}(t) \\
& =: \beta_{n, k} p_{n+m-1, k+m+r-1}(t)
\end{aligned}
$$

and $\alpha_{n, k} \beta_{n, k} \leq C$, we get $\varphi^{2 r \lambda}(x) I \leq\left\|\varphi^{2 r \lambda} f^{(2 r)}\right\|$. This completes the proof of Lemma 5 .

Lemma 6 ([8]) For $0<t<\frac{1}{16 r}, \frac{r t}{2}<x<1-\frac{r t}{2}, 0 \leq \beta \leq 2 r$, we have

$$
\int \cdots \int_{-\frac{t}{2}}^{\frac{t}{2}} \varphi^{-\beta}\left(x+u_{1}+\cdots+u_{2 r}\right) d u_{1} \cdots d u_{2 r} \leq C t^{2 r} \varphi^{-\beta}(x) .
$$

\section{Proof of the theorems}

In this section we will give the proof of Theorem 1 and Theorem 2.

Proof of Theorem 1 By the definition of $K_{\varphi^{\lambda}}\left(f, t^{2 r}\right)$ and the equivalence between $\omega_{\varphi^{\lambda}}^{2 r}(f, t)$ and $K_{\varphi^{\lambda}}\left(f, t^{2 r}\right)$, for the fixed $n$ and $x$, we can choose $g=g_{n, x}$ such that

$$
\|f-g\|+\frac{1}{n}\left\|\varphi^{2 \lambda} g^{\prime \prime}\right\| \leq C \omega_{\varphi^{\lambda}}^{2}\left(f, \frac{1}{\sqrt{n}}\right) .
$$

We know that

$$
\left|M_{n, m}(f, x)-f(x)\right| \leq 2\|f-g\|+\left|M_{n, m}(g, x)-g(x)\right|,
$$


Li Journal of Inequalities and Applications 2014, 2014:106

Page 5 of 6

http://www.journalofinequalitiesandapplications.com/content/2014/1/106

and we have to estimate the second term on the right side of (3.2). By Taylor's formula, $g(t)=g(x)+g^{\prime}(x)(t-x)+\int_{x}^{t}(t-u) g^{\prime \prime}(u) d u$, and Lemma 3 we have

$$
\begin{aligned}
\left|M_{n, m}(g, x)-g(x)\right| & \leq\left|g^{\prime}(x)\right|\left|M_{n, m}((t-x), x)\right|+\left|M_{n, m}\left(\int_{x}^{t}(t-u) g^{\prime \prime}(u) d u, x\right)\right| \\
& \leq C \frac{1}{n}\left|g^{\prime}(x)\right|+\left|M_{n, m}\left(\int_{x}^{t}(t-u) g^{\prime \prime}(u) d u, x\right)\right| \\
& =: I_{1}+I_{2} .
\end{aligned}
$$

We consider $I_{1}$ first. For $0 \leq x \leq \frac{1}{2}$,

$$
\left|g^{\prime}(x)-g^{\prime}\left(\frac{1}{2}\right)\right|=\left|\int_{x}^{\frac{1}{2}} g^{\prime \prime}(u) d u\right| \leq\left\|x^{\lambda} g^{\prime \prime}\right\| \int_{x}^{\frac{1}{2}} \frac{1}{u^{\lambda}} d u \leq C\left\|\varphi^{2 \lambda} g^{\prime \prime}\right\|,
$$

together with $\left|g^{\prime}\left(\frac{1}{2}\right)\right| \leq C\left(\left\|g^{\prime \prime}\right\|_{L_{\infty}\left[\frac{1}{4}, \frac{3}{4}\right]}+\|g\|_{L_{\infty}\left[\frac{1}{4}, \frac{3}{4}\right]}\right) \leq C\left(\left\|\varphi^{2 \lambda} g^{\prime \prime}\right\|+\|g\|\right)$, one has

$$
\left|g^{\prime}(x)\right| \leq C\|g\|+\left\|\varphi^{2 \lambda} g^{\prime \prime}\right\| \text {. }
$$

It is similar for $\frac{1}{2}<x \leq 1$.

Now we address $I_{2}$. By the process of (9.6.1) in [7]

$$
\left|R_{2 r}(f, u, x)\right| \leq \frac{|u-x|^{2 r-1}}{\varphi^{2 r}(x)}\left|\int_{u}^{x} \varphi^{2 r}(v)\right| f^{(2 r)}(v)|d v|
$$

we get $\frac{|t-u|}{\varphi^{2 \lambda}(u)} \leq \frac{|t-x|}{\varphi^{2 \lambda}(x)}$, and combining with (2.1) we deduce

$$
I_{2} \leq \frac{\left\|\varphi^{2 \lambda} g^{\prime \prime}\right\|}{\varphi^{2 \lambda}(x)} M_{n, m}\left((t-x)^{2}, x\right) \leq C \frac{\left\|\varphi^{2 \lambda} g^{\prime \prime}\right\|}{\varphi^{2 \lambda}(x)} \frac{\varphi^{2}(x)}{n} \leq C \frac{1}{n}\left\|\varphi^{2 \lambda} g^{\prime \prime}\right\| .
$$

By (3.2)-(3.5), we complete the proof of Theorem 1.

Proof of Theorem 2 For convenience let $\gamma_{n, \lambda}(x)=n^{-\frac{1}{2}} \delta_{n}^{1-\lambda}(x)$. If $M_{n, m}(f, x)-f(x)=$ $O\left(\gamma_{n, \lambda}^{\alpha}(x)\right)$, for every $n: n>2 r$, we have

$$
\begin{aligned}
\left|\triangle_{t \varphi^{\lambda}(x)}^{2 r} f(x)\right| \leq & \left|\triangle_{t \varphi^{\lambda}(x)}^{2 r}\left(M_{n, m}(f, x)-f(x)\right)\right|+\left|\triangle_{t \varphi^{\lambda}(x)}^{2 r} M_{n, m}(f, x)\right| \\
\leq & C \gamma_{n, \lambda}^{\alpha}(x)+\int \cdots \int_{-\frac{t \varphi^{\lambda}(x)}{2}}^{\frac{t \varphi^{\lambda}(x)}{2}}\left|M_{n, m}^{(2 r)}\left(f, x+\sum_{j=1}^{2 r} u_{j}\right)\right| d u_{1} \cdots d u_{2 r} \\
\leq & C \gamma_{n, \lambda}^{\alpha}(x)+\int \cdots \int_{-\frac{t \varphi^{\lambda}(x)}{2}}^{\frac{t \varphi^{\lambda}(x)}{2}}\left|M_{n, m}^{(2 r)}\left(f-g, x+\sum_{j=1}^{2 r} u_{j}\right)\right| d u_{1} \cdots d u_{2 r} \\
& +\int \ldots \int_{-\frac{t \varphi^{\lambda}(x)}{2}}^{\frac{t \varphi^{\lambda}(x)}{2}}\left|M_{n, m}^{(2 r)}\left(g, x+\sum_{j=1}^{2 r} u_{j}\right)\right| d u_{1} \cdots d u_{2 r} \\
:= & C \gamma_{n, \lambda}^{\alpha}(x)+J_{1}+J_{2} .
\end{aligned}
$$


Combining Lemma 4, Lemma 5, and Lemma 6, we have

$$
\begin{aligned}
& J_{1} \leq C t^{2 r} \gamma_{n, \lambda}^{-2 r}(x)\|f-g\|, \\
& J_{2} \leq C t^{2 r}\left\|\varphi^{2 r \lambda} g^{(2 r)}\right\| .
\end{aligned}
$$

Utilizing (3.6), (3.7), and (3.8), choosing the appropriate $g$, we obtain

$$
\left|\triangle_{t \varphi^{\lambda}(x)}^{2 r} f(x)\right| \leq C\left(\gamma_{n, \lambda}^{\alpha}(x)+t^{2 r} \gamma_{n, \lambda}^{-2 r}(x) \omega_{\varphi^{\lambda}}^{2 r}\left(f, \gamma_{n, \lambda}(x)\right)\right) .
$$

For every fixed $h: 0<h<\frac{1}{16 r}$ and every $x: x \geq r t$, we can choose $n$ such that $\gamma_{n, \lambda}(x) \leq h<$ $2 \gamma_{n, \lambda}(x)$. Then

$$
\left|\triangle_{t \varphi^{\lambda}(x)}^{2 r} f(x)\right| \leq C\left(h^{\alpha}+\left(\frac{t}{h}\right)^{2 r} \omega_{\varphi^{\lambda}}^{2 r}(f, h)\right) .
$$

So,

$$
\omega_{\varphi^{\lambda}}^{2 r}(f, t) \leq C\left(h^{\alpha}+\left(\frac{t}{h}\right)^{2 r} \omega_{\varphi^{\lambda}}^{2 r}(f, h)\right)
$$

which yields the assertion of Theorem 2 by the Berens-Lorentz lemma.

\section{Competing interests}

The author declares that they have no competing interests.

Received: 13 June 2013 Accepted: 20 February 2014 Published: 04 Mar 2014

\section{References}

1. Durrmeyer, JL: Une formule d'inversion de la transformée de Laplace: applications à la théorie des moments. Thèse de 3e cycle, Faculte des Science de l'Université de Paris (1967)

2. Derriennic, MM: Sur l'approximation de fonctions intégrables sur $[0,1]$ par des polynômes de Bernstein modifiés. J. Approx. Theory 31, 323-343 (1981)

3. Zeng, $\mathrm{XM}, \mathrm{Chen}, \mathrm{W}$ : On the rate of convergence of the generalized Durrmeyer type operators for functions of bounded variation. J. Approx. Theory 102, 1-12 (2000)

4. Heiner, G, Daniela, K, loan, R: The genuine Bernstein-Durrmeyer operators revisited. Results Math. 62, 295-310 (2012). doi:10.1007/s00025-012-0287-1

5. Gupta, V: Approximation properties by Bernstein-Durrmeyer type operators. Complex Anal. Oper. Theory 7, 363-374 (2013). doi:10.1007/s11785-011-0167-9

6. Gupta, V, López-Moreno, AJ, Latorre-Palacios, JM: On simultaneous approximation of the Bernstein Durrymeyer operators. Appl. Math. Comput. 213, 112-120 (2009)

7. Ditzian, Z, Totik, V: Moduli of Smoothness. Springer, New York (1987)

8. Guo, SS, Liu, LX, Qi, QL: Pointwise estimate for linear combinations of Bernstein-Kantorovich operators. J. Math. Anal. Appl. 265, 135-147 (2002) 